\title{
Rock Characterization Through Physical Properties and Their Relationship to Simple Compressive Strength
}

\section{Caracterización de la Roca Mediante Propiedades Físicas y Su Relación con la Resistencia a la Compresión Simple}

\author{
Patricio Feijoo C. and Elizabeth Brito V.
}

I International Congress of Science and Technology Morona Santiago-CICTMS 2020

Corresponding Author:

Patricio Feijoo C.

pfeijoo@uazuay.edu.ec

Published: 29 August 2021

Production and Hosting by Knowledge E

(c) Patricio Feijoo C. and Elizabeth Brito V.. This article is distributed under the terms of the Creative Commons Attribution License, which permits unrestricted use and redistribution provided that the original author and source are credited.

\section{S OPEN ACCESS}

Universidad del Azuay, Facultad de Ciencia y Tecnología, Escuela de Ingeniería en Minas, Cuenca, Ecuador

\section{Abstract}

This work proposes a simple methodology and practical application in the field for the approximate determination of the Unconfined Compressive Strength (UCS) in rocks, property or characteristic that is important in mining, since through it analyzes are carried out to the assessment of security and stability factors and/or possible fortification systems in the works or mining structures, in addition to the characterization of the UCS is also influential in the use of explosives for the exploitation or extraction of materials from a quarry or mine. This estimate is proposed based on the determination of the following three properties of the rock, which in this investigation we call density, porosity and absorption 'in mine'. These physical properties can be obtained in a simple, but methodical way and in this work, tests have been carried out on the same material or rock from the Cojitambo area, Cañar province (Ecuador) and on the basis of 60 samples or test tubes. The results obtained allow a correlation between the properties described above and the UCS, in addition to a calculation methodology for the proposed objective.

Keywords: compression, rock, density, porosity, absorption, fortification.

\section{Resumen}

En este trabajo se propone una metodología sencilla y de aplicación práctica en campo para la determinación aproximada de la Resistencia a la Compresión Simple (RCS) en rocas, propiedad o característica que es importante en minería, ya que mediante la misma, se ejecutan análisis para la valoración de factores de seguridad y estabilidad y/o posibles sistemas de fortificación en las obras o estructuras mineras, a más de que la caracterización de la RCS es también influyente en el uso de explosivos para la explotación o extracción de materiales de una cantera o mina. Esta estimación se la propone en base a la determinación de las siguientes tres propiedades de la roca, que en esta investigación las denominamos densidad, porosidad y absorción 'en mina'. Estas propiedades físicas se las puede obtener de una forma simple, pero metódica y en este trabajo se han ejecutado ensayos sobre un mismo material o roca proveniente de la zona de Cojitambo, provincia del Cañar (Ecuador) y sobre una base de 60 muestras o probetas. Los resultados obtenidos permiten una correlación entre las propiedades antes descritas y la RCS, a más que se ha estructurado una metodología de cálculo para el objetivo planteado.

How to cite this article: Patricio Feijoo C., Elizabeth Brito V. (2021). Rock Characterization Through Physical Properties and Their Relationship to Simple 


\section{Introducción}

En el desarrollo de la explotación de minas y canteras es importante determinar la resistencia a la compresión simple de las rocas (RCS) ya que mediante este parámetro se pueden desarrollar clasificaciones de los macizos rocosos, como la del Rock Mass Rating (RMR) o Índice $Q$, con las cuales se determina la estabilidad de las estructuras mineras, tanto a cielo abierto como en subterráneo. La resistencia de una roca o de sedimentos no bien consolidados está influida por la mineralogía de sus partículas y por el contacto que hay entre ellas [1]. Pero muchas veces debido a las condiciones en las cuales se encuentran las labores mineras, resulta complicado y a veces casi imposible enviar muestras de roca a laboratorios para determinar la RCS, y en estos tiempos es la problemática de los encargados de evaluar la estabilidad de las estructuras en el campo, por lo que se presenta la imperiosa necesidad de presentar alternativas in situ para la valoración de la RCS. En este trabajo se propone determinar propiedades físicas de las rocas y correlacionarlas con la RCS, para de esta manera obtener de una manera aproximada dicho parámetro. En la Mecánica de Rocas, que es la encargada de estudiar las propiedades de las rocas y de los macizos rocosos, es muy importante definir algunas de ellas, que sin restar importancia a otras, se consideran prioritarias. El comportamiento de una masa o macizo de roca in situ es diferente de un material rocoso, debido a que el material rocoso es mucho más fuerte y un macizo rocoso presenta casi siempre sistemas de debilidades estructurales llamadas diaclasas (fracturas, fisuras, juntas, discontinuidades, fallas de varios tamaños). Prácticamente todas las rocas que forman los kilómetros de la corteza terrestre están atravesadas por fisuras y grietas de corta extensión [2].

En este punto cabe analizar cada una de las propiedades de las rocas que se verán involucradas en las diferentes propuestas de correlación de este trabajo, tomando en consideración que la heterogeneidad de los materiales o rocas usados siempre puede ser un limitante para su análisis. También, incluso en rocas aparentemente isótropas y homogéneas, entendiéndose como homogeneidad si dos muestras cualesquiera de una masa rocosa del mismo volumen e igualmente orientadas son idénticas desde todos los puntos de vista [3], las propiedades pueden variar según el grado de cementación o variaciones en la composición mineralógica.

\section{Metodología}

Se denomina Resistencia a la Compresión Simple de una roca (RCS) al esfuerzo medido sobre la misma de una manera técnica. Esta valoración o parámetro se debe a la necesidad de emplear clasificaciones de macizos rocosos. Estas clasificaciones son conocidas como clasificaciones geomecánicas y derivan de la diferencia existente entre las propiedades de la roca por su naturaleza y las del macizo, que presenta diferentes grados de fracturación y meteorización. Las clasificaciones geomecánicas son sistemas de valoración del comportamiento del terreno rocoso. Se basan en calificar numéricamente las propiedades y características específicas de la roca en un emplazamiento determinado y posteriormente obtener una calificación final como 
la suma de las valoraciones parciales. En la actualidad las clasificaciones geomecánicas se han generalizado y son ampliamente utilizadas, tanto en las fases de diseño como en las fases de ejecución, en todo tipo de obras en macizos rocosos. Es importante, por tanto, conocer las limitaciones y dificultades que presenta cada una de las clasificaciones [4]. Las diferentes clasificaciones toman en cuenta un parámetro fundamental, la Resistencia a la Compresión Uniaxial o Simple de la Roca. La RCS es una medida de resistencia de la matriz rocosa, la cual representa el esfuerzo de compresión axial máximo que puede soportar una muestra de material antes de fracturarse.

Este ensayo sirve para determinar la resistencia a compresión de una probeta cilíndrica de roca de altura entre el doble y el triple del diámetro [5]. Normalmente estas probetas se obtienen a partir de testigos de perforación. También se pueden obtener muestras a partir de bloques de roca; la extracción de estos bloques en la mina o en la obra se debe llevar a cabo sin voladuras, ya que éstas pueden generar en la roca nuevas microfisuras o aumentar las existentes, lo cual se traduciría en una pérdida de resistencia de las probetas que se obtengan de ellos. Averiguar la resistencia a compresión simple de una roca es importante porque permite clasificar la roca según su resistencia, es un parámetro importante en los criterios de rotura más utilizados (Mohr-Coulomb y Hoek-Brown) [6]. La Tabla 1 muestra una clasificación de las rocas y la resistencia a la compresión según la ISRM (International Society for Rock Mechanics) de 1981 [7].

\section{Table 1}

Clasificación de la roca en base a la resistencia a la compresión según la ISRM.

Descripción
Extremadamente blanda
Muy blanda
Blanda
Moderadamente blanda
Dura
Muy dura
Extremadamente dura

Resistencia a la Comprensión Simple (MPa)
$<1$
1 a 5
5 a 25
25 a 50
50 a 100
100 a 250
$>250$

Fuente: ISRM 1981.

Las rocas presentan relaciones lineales y/o no lineales entre las fuerzas aplicadas y las deformaciones producidas, obteniéndose diferentes modelos de curvas de tensión contra deformación para distintos tipos de rocas [8].

La densidad es una propiedad elemental y fundamental de los materiales, relacionada con la naturaleza de sus constituyentes y la existencia de espacios vacíos entre ellos. La densidad $(\rho)$ se define como la masa (M) por unidad de volumen (V), y se expresa en $\mathrm{kg} / \mathrm{m}^{3}$.

Conocida la masa y el volumen de una muestra rocosa se determina de forma inmediata su densidad. En los materiales porosos tanto la masa como el volumen admiten ciertas matizaciones y, en consecuencia, se pueden establecer distintos tipos de densidad. Fundamentalmente se distingue dos: 'densidad de los granos minerales' y 


\section{Table 2}

Datos y resultados.

\begin{tabular}{|c|c|c|c|c|c|c|c|c|c|c|c|}
\hline & Mi & a & b & c & $\begin{array}{l}\text { Densidad } \\
\text { en mina }\end{array}$ & Fuerza & RCS & Mf & $\begin{array}{l}\text { Porosidad } \\
\text { en mina }\end{array}$ & Ma & $\begin{array}{l}\text { Absorción } \\
\text { en mina }\end{array}$ \\
\hline & g & $\mathrm{cm}$ & $\mathrm{cm}$ & $\mathrm{cm}$ & $\mathrm{Kg} / \mathrm{m}^{3}$ & Kg & MPa & g & $\%$ & g & $\%$ \\
\hline 1 & 725.40 & 4.90 & 12.20 & 5.00 & 2426.90 & 11445.00 & 45.80 & 721.80 & 1.20 & 738.20 & 4.28 \\
\hline 2 & 776.40 & 5.20 & 12.30 & 5.00 & 2427.77 & 19748.00 & 74.46 & 772.90 & 1.09 & 793.60 & 5.38 \\
\hline 3 & 761.60 & 5.20 & 11.90 & 4.90 & 2511.77 & 13794.00 & 53.08 & 757.80 & 1.25 & 774.20 & 4.16 \\
\hline 4 & 779.20 & 5.10 & 12.20 & 5.10 & 2455.55 & 12626.00 & 47.59 & 775.60 & 1.13 & 798.20 & 5.99 \\
\hline 5 & 778.60 & 5.00 & 12.10 & 5.10 & 2523.42 & 19106.00 & 73.46 & 774.90 & 1.20 & 794.00 & 4.99 \\
\hline 6 & 779.60 & 5.10 & 12.30 & 5.20 & 2389.97 & 18713.00 & 69.18 & 775.70 & 1.20 & 792.10 & 3.83 \\
\hline 7 & 714.40 & 5.00 & 11.90 & 4.80 & 2501.40 & 9274.00 & 37.88 & 711.10 & 1.16 & 726.50 & 4.24 \\
\hline 8 & 756.20 & 5.00 & 12.10 & 5.10 & 2450.82 & 12551.00 & 48.25 & 752.70 & 1.13 & 772.90 & 5.41 \\
\hline 9 & 776.60 & 5.10 & 12.30 & 5.10 & 2427.46 & 14429.00 & 54.39 & 772.70 & 1.22 & 791.70 & 4.72 \\
\hline 10 & 784.80 & 5.00 & 12.20 & 5.20 & 2474.15 & 12629.00 & 47.62 & 781.10 & 1.17 & 801.00 & 5.11 \\
\hline 11 & 739.80 & 4.90 & 11.90 & 5.00 & 2537.47 & 13055.00 & 52.24 & 735.90 & 1.34 & 748.50 & 2.98 \\
\hline 12 & 727.20 & 5.00 & 12.10 & 5.00 & 2403.97 & 14651.00 & 57.45 & 725.40 & 0.60 & 730.10 & 0.96 \\
\hline 13 & 764.60 & 5.10 & 12.10 & 5.20 & 2382.73 & 16221.00 & 59.97 & 760.70 & 1.22 & 776.80 & 3.80 \\
\hline 14 & 763.70 & 5.10 & 12.00 & 5.10 & 2446.82 & 14210.00 & 53.56 & 760.00 & 1.19 & 777.10 & 4.29 \\
\hline 15 & 771.90 & 5.10 & 12.10 & 5.10 & 2452.65 & 18191.00 & 68.57 & 768.30 & 1.14 & 788.70 & 5.34 \\
\hline 16 & 757.30 & 5.00 & 12.10 & 5.10 & 2454.38 & 12634.00 & 48.57 & 753.60 & 1.20 & 773.50 & 5.25 \\
\hline 17 & 735.00 & 5.20 & 11.90 & 5.10 & 2328.99 & 14413.00 & 53.28 & 733.00 & 0.63 & 739.20 & 1.33 \\
\hline 18 & 767.50 & 5.00 & 12.30 & 5.30 & 2354.66 & 20735.00 & 76.71 & 763.30 & 1.29 & 776.20 & 2.67 \\
\hline 19 & 761.10 & 5.10 & 12.20 & 5.00 & 2446.48 & 19641.00 & 75.51 & 757.30 & 1.22 & 773.00 & 3.83 \\
\hline 20 & 760.40 & 5.10 & 12.00 & 5.00 & 2484.97 & 9209.00 & 35.41 & 756.60 & 1.24 & 777.20 & 5.49 \\
\hline 21 & 743.80 & 4.90 & 12.30 & 5.00 & 2468.23 & 17269.00 & 69.10 & 740.10 & 1.23 & 758.30 & 4.81 \\
\hline 22 & 766.40 & 5.00 & 12.00 & 5.00 & 2554.67 & 16259.00 & 63.76 & 762.60 & 1.27 & 778.40 & 4.00 \\
\hline 23 & 778.50 & 5.20 & 12.00 & 5.20 & 2399.22 & 12964.00 & 47.00 & 774.90 & 1.11 & 794.50 & 4.93 \\
\hline 24 & 764.10 & 5.10 & 12.00 & 5.10 & 2448.10 & 6577.00 & 24.79 & 760.50 & 1.15 & 774.80 & 3.43 \\
\hline 25 & 785.90 & 5.00 & 12.30 & 5.10 & 2505.66 & 9757.00 & 37.51 & 782.40 & 1.12 & 788.80 & 0.92 \\
\hline 26 & 774.20 & 5.40 & 12.10 & 5.00 & 2369.76 & 13544.00 & 49.18 & 771.40 & 0.86 & 778.50 & 1.32 \\
\hline 27 & 759.60 & 5.10 & 11.90 & 5.10 & 2454.13 & 15732.00 & 59.30 & 756.80 & 0.90 & 764.10 & 1.45 \\
\hline 28 & 792.80 & 5.20 & 12.30 & 5.00 & 2479.05 & 13560.00 & 51.13 & 789.50 & 1.03 & 798.10 & 1.66 \\
\hline 29 & 798.30 & 5.50 & 12.10 & 5.00 & 2399.10 & 11662.00 & 41.58 & 794.40 & 1.17 & 799.90 & 0.48 \\
\hline 30 & 801.70 & 5.30 & 12.20 & 5.00 & 2479.74 & 24060.00 & 89.01 & 798.40 & 1.02 & 805.90 & 1.30 \\
\hline 31 & 772.80 & 5.00 & 12.00 & 5.10 & 2525.49 & 22399.00 & 86.12 & 769.20 & 1.18 & 776.60 & 1.24 \\
\hline 32 & 766.70 & 4.90 & 11.90 & 5.20 & 2528.59 & 12683.00 & 48.80 & 763.80 & 0.96 & 771.80 & 1.68 \\
\hline 33 & 779.30 & 5.20 & 12.00 & 5.00 & 2497.76 & 14126.00 & 53.27 & 775.70 & 1.15 & 782.50 & 1.03 \\
\hline 34 & 769.50 & 5.00 & 12.10 & 5.00 & 2543.80 & 19890.00 & 78.00 & 766.20 & 1.09 & 774.00 & 1.49 \\
\hline 35 & 769.30 & 5.30 & 12.20 & 5.00 & 2379.52 & 20901.00 & 77.33 & 764.80 & 1.39 & 771.80 & 0.77 \\
\hline 36 & 800.20 & 5.30 & 12.50 & 5.10 & 2368.33 & 7207.00 & 26.14 & 795.50 & 1.39 & 803.00 & 0.83 \\
\hline 37 & 784.50 & 5.10 & 12.20 & 5.40 & 2334.90 & 22323.00 & 79.47 & 780.80 & 1.10 & 788.80 & 1.28 \\
\hline 38 & 793.10 & 5.20 & 12.10 & 5.10 & 2471.55 & 23994.00 & 88.70 & 789.20 & 1.22 & 796.00 & 0.90 \\
\hline
\end{tabular}


Table 2

Continued

\begin{tabular}{|c|c|c|c|c|c|c|c|c|c|c|c|}
\hline & Mi & $\mathbf{a}$ & b & c & $\begin{array}{l}\text { Densidad } \\
\text { en mina }\end{array}$ & Fuerza & RCS & Mf & $\begin{array}{l}\text { Porosidad } \\
\text { en mina }\end{array}$ & Ma & $\begin{array}{l}\text { Absorción } \\
\text { en mina }\end{array}$ \\
\hline & g & $\mathrm{cm}$ & $\mathrm{cm}$ & $\mathrm{cm}$ & $\mathrm{Kg} / \mathbf{m}^{3}$ & Kg & $\mathrm{MPa}$ & g & $\%$ & g & $\%$ \\
\hline & 802.80 & 5.10 & 12.40 & 5.10 & 2489.12 & 18091.00 & 68.19 & 798.20 & 1.43 & 804.40 & 0.50 \\
\hline & 774.40 & 5.00 & 12.10 & 5.30 & 2415.09 & 21607.00 & 79.94 & 770.80 & 1.12 & 777.90 & 1.09 \\
\hline 41 & 785.90 & 5.10 & 12.10 & 5.00 & 2547.08 & 21952.00 & 84.40 & 780.10 & 1.88 & 786.90 & 0.32 \\
\hline & 795.20 & 5.00 & 12.20 & 5.10 & 2556.09 & 19160.00 & 73.66 & 791.90 & 1.06 & 799.70 & 1.45 \\
\hline & 771.30 & 5.10 & 12.10 & 5.00 & 2499.76 & 19667.00 & 75.61 & 767.80 & 1.13 & 775.50 & 1.36 \\
\hline & 794.10 & 5.00 & 12.30 & 5.30 & 2436.26 & 23744.00 & 87.84 & 790.20 & 1.20 & 797.30 & 0.98 \\
\hline 45 & 786.90 & 5.20 & 12.10 & 5.20 & 2405.07 & 22434.00 & 81.34 & 783.10 & 1.16 & 789.50 & 0.79 \\
\hline & 727.40 & 5.00 & 12.00 & 5.00 & 2424.67 & 8793.00 & 34.48 & 722.00 & 1.80 & 733.00 & 1.87 \\
\hline 47 & 711.30 & 5.00 & 11.80 & 5.00 & 2411.19 & 17345.00 & 68.02 & 708.00 & 1.12 & 723.30 & 4.07 \\
\hline 8 & 720.80 & 4.90 & 12.00 & 5.00 & 2451.70 & 22725.00 & 90.94 & 717.10 & 1.26 & 723.60 & 0.95 \\
\hline 49 & 725.70 & 5.00 & 12.00 & 5.00 & 2419.00 & 15251.00 & 59.81 & 722.20 & 1.17 & 730.40 & 1.57 \\
\hline 0 & 699.30 & 5.00 & 11.90 & 5.00 & 2350.59 & 7287.00 & 28.58 & 695.20 & 1.38 & 710.90 & 3.90 \\
\hline 51 & 700.00 & 5.00 & 11.70 & 5.00 & 2393.16 & 10087.00 & 39.56 & 695.20 & 1.64 & 711.90 & 4.07 \\
\hline & 708.60 & 5.00 & 11.90 & 5.00 & 2381.85 & 13945.00 & 54.69 & 705.10 & 1.18 & 718.00 & 3.16 \\
\hline 53 & 693.00 & 5.00 & 11.90 & 4.70 & 2478.10 & 16250.00 & 67.79 & 691.30 & 0.61 & 704.00 & 3.93 \\
\hline 54 & 725.60 & 4.90 & 12.00 & 5.00 & 2468.03 & 13220.00 & 52.90 & 719.90 & 1.94 & 728.10 & 0.85 \\
\hline 55 & 714.20 & 5.00 & 11.90 & 5.00 & 2400.67 & 12149.00 & 47.64 & 711.50 & 0.91 & 726.00 & 3.97 \\
\hline 56 & 707.70 & 5.00 & 11.90 & 4.90 & 2427.37 & 19256.00 & 77.05 & 702.30 & 1.85 & 711.00 & 1.13 \\
\hline & 710.10 & 4.90 & 11.90 & 5.00 & 2435.60 & 13256.00 & 53.05 & 709.20 & 0.31 & 721.00 & 3.74 \\
\hline & 709.60 & 5.00 & 12.00 & 5.00 & 2365.33 & 15665.00 & 61.43 & 705.30 & 1.43 & 722.00 & 4.13 \\
\hline 59 & 701.80 & 4.90 & 11.90 & 4.90 & 2456.26 & 21191.00 & 86.53 & 698.50 & 1.15 & 713.00 & 3.92 \\
\hline 0 & 713.60 & 5.00 & 11.90 & 4.90 & 2447.61 & 20340.00 & 81.39 & 710.20 & 1.17 & 725.00 & 3.91 \\
\hline
\end{tabular}

Fuente: Autores.

'densidad de la roca seca'. También se consideran otros tipos de densidad, obtenidos a partir de distintos ensayos, como la 'densidad de la roca húmeda' (para un determinado contenido en humedad) y la 'densidad corregida' o 'densidad del esqueleto' (cuando el volumen de roca no incluye los poros abiertos pero incluye los cerrados) [9].

La obtención de la masa de la muestra de una roca no es un problema, únicamente es necesario que esté seca, por lo que los distintos métodos se diferencian en el procedimiento seguido para determinar el volumen. Un método sencillo, es el de generar prismas de roca con medidas más o menos iguales y mediante el uso de un calibrador se determina el volumen de las mismas.

La porosidad en rocas es definida como la razón entre el volumen de los espacios vacíos entre la roca y el total volumen aparente de la roca, también se refiere a la probabilidad de encontrar vacíos en el volumen total [10]. 
Así también el contenido de humedad presente en una roca es la relación existente entre el agua contenida en los poros de la roca y la masa de las partículas de la roca misma. Si el sólido se encuentra directamente en contacto con el agua líquida, todos estos poros capilares son rápidamente saturados de agua, hablándose entonces de absorción capilar [11].

Esta propiedad, será para alguna roca mayor que para otras, y en este punto podemos hablar de la velocidad con la cual absorbe el agua la probeta, a través de su capilaridad, propiedad denominada sorptividad [12].

La base de cualquier trabajo es la planificación de las actividades a realizar. Para cumplir con el objetivo disponemos de una serie de herramientas, unas para aplicar en campo y otras en laboratorio.

Para el desarrollo de la propuesta se inició con la obtención de muestras de un sector denominado Cojitambo, presenta una morfología de tipo multiforme, es una formación volcánica en la provincia del Cañar (Ecuador) [13]. Estas muestras se tomaron de afloramientos, pero trabajando en los mismos para que la matriz rocosa extraída sea sana y no contenga ningún tipo de alteración o descomposición. Es muy importante que las muestras tengan superficies frescas y limpias, que nos permita realizar una valoración correcta del estado de la roca a la cual se procede a realizar los ensayos [14]. Realizada la toma de muestras se las preparó para la elaboración de probetas, en lo posible de iguales dimensiones. Las probetas preparadas tuvieron las siguientes dimensiones aproximadamente: $5 \times 5 \times 12 \mathrm{~cm}$ y en total se elaboraron 60 probetas.

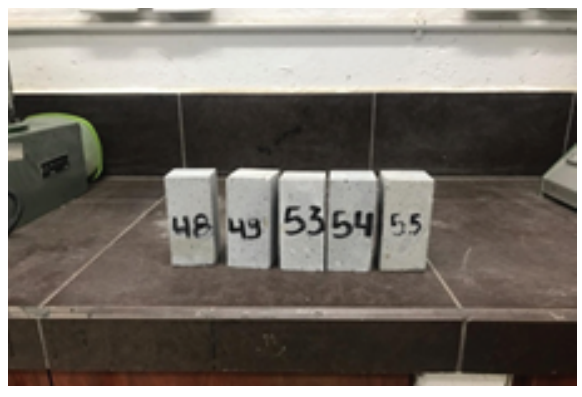

\section{Figure 1}

Muestras o probetas de roca.

En la primera fase se ejecutaron las pruebas para la obtención de lo que denominamos densidad en mina, para lo cual con una balanza de precisión se obtiene la masa y con sus dimensiones se obtuvieron sus respectivos volúmenes [15].

$$
\rho=\frac{M i}{V i}
$$

donde:

$\rho:$ densidad en mina en $\frac{K g}{m^{3}}$;

Mi: masa inicial en $\mathrm{Kg}$;

Vi: volumen inicial en $\mathrm{m}^{3}$.

En una segunda parte, luego de haber determinado masa y volumen de cada probeta, las mismas se proceden a introducir en un horno convencional, a temperatura constate 
de $75^{\circ} \mathrm{C}$, por el tiempo que sea necesario hasta que la masa de cada probeta se mantenga constante, es decir hasta que no se produzca más perdida de humedad de la misma. A esta prueba o etapa la denominamos porosidad en mina de la roca. El tiempo necesario para esta etapa estuvo en el orden de 4 a 5 días.

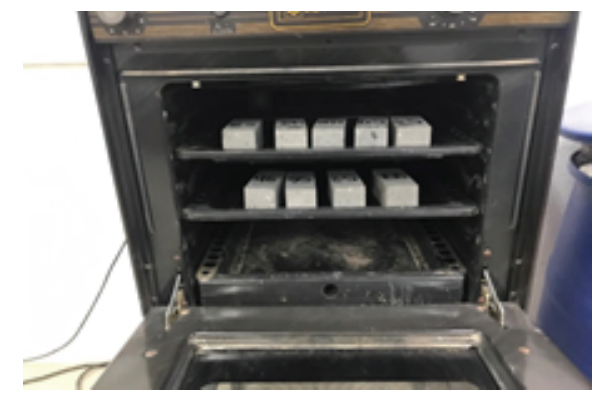

Figure 2

Probetas de roca en horno para secado.

La porosidad en mina de las diferentes probetas la obtenemos mediante la siguiente relación [15]:

$$
\eta=\frac{M i-M f}{V i} \times 100,
$$

donde:

ๆ: porosidad en mina en \%;

Mf: masa al fin del secado en $\mathrm{Kg}$.

Como tercera etapa, luego de que las probetas se han estabilizado en su masa y luego de haberlas dejando a temperatura ambiente por al menos 7 días, se procede a ejecutar la prueba que hemos denominado absorción en mina de la roca, la cual consiste en introducir la probeta en un vaso de precipitado de capacidad de un litro, conteniendo agua hasta los $500 \mathrm{cc}$. Esta prueba se puede decir que es con sorptividad constante, ya que se introduce la probeta por una hora exacta y luego de eso se obtiene la nueva masa de la probeta.

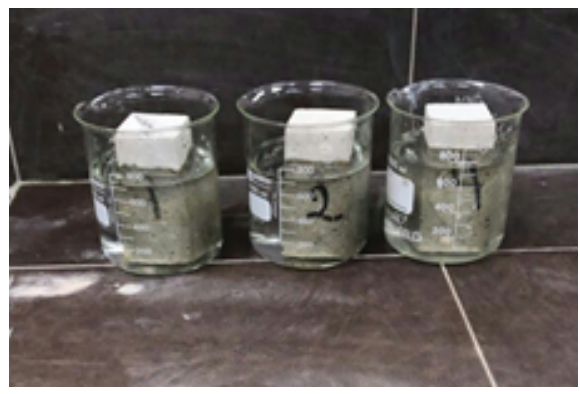

\section{Figure 3}

Probetas de roca en vaso para absorción de agua.

Para la determinación de la absorción en mina utilizamos la siguiente relación [15]:

$$
\omega=\frac{M a-M i}{V i} \times 100,
$$


donde:

$\omega:$ absorción en mina en \%;

Ma: masa final con agua en $\mathrm{Kg}$.

Obtenidos todos estos resultados, se procede nuevamente a mantener las probetas a temperatura ambiente por otros 7 días, hasta que las mismas vuelvan a sus condiciones originales de ser posible.

Finalmente se las lleva al laboratorio para la determinación de la resistencia a la compresión. Para la ejecución de los ensayos se utiliza el equipo apropiado. Esta máquina es una prensa Humboldt que tiene facultades para someter materiales a ensayos de tensión y compresión. La presión se logra mediante placas o mandíbulas accionadas por tornillos o sistema hidráulico.

La máquina de ensayos tiene como función comprobar la resistencia de diversos tipos de materiales, para esto posee un sistema que aplica cargas controladas sobre una probeta (modelo de dimensiones preestablecidas) y mide en forma gráfica la deformación, y la carga al momento de su ruptura (Figura 4).

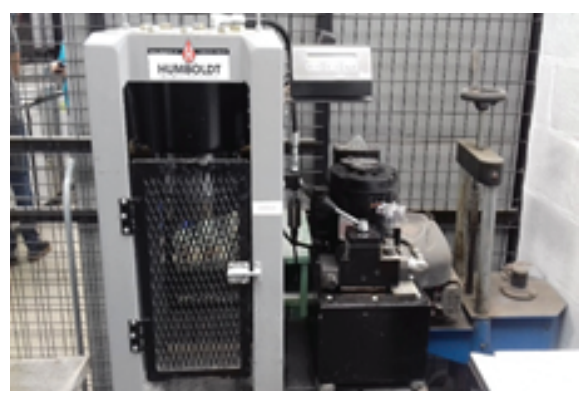

Figure 4

Máquina de compresión Humboldt.

Las recomendaciones para la aplicación de la máquina de compresión son: (a) Adecuado para cilindros, cubos, vigas y núcleos de mezclas. (b) Rango de prueba de 2500 a 250000 lb (11 a 1112 KN) con una precisión de $\pm 0.5 \%$ de la carga indicada. (c) La configuración estándar incluye platos para probar cilindros de 6"×12" $(150 \times 300$ mm) y se procede a realizar los cálculos de carga máxima y sección transversal mediante las normas ASTM. C42-68 para corrección de la resistencia a la compresión simple [16].

\section{Desarrollo y Discusión}

Luego de evaluar los resultados, Tabla 2 , se ha establecido que la densidad en mina presenta una serie de valores que varían entre los 2329 a los $2556 \mathrm{Kg} / \mathrm{m}^{3}$, pero si establecemos subconjuntos, en este caso 6 debido a que se trabajó sobre 60 muestras, la mayor cantidad de muestras se ubican en el subconjunto de 2443 a $2480 \mathrm{Kg} / \mathrm{m}^{3}$, y este rango de valores nos presenta en promedio una RCS de $64 \mathrm{MPa}$ (Figura 5).

Analizados los datos de porosidad en mina, Figura 6 , estos presentan valores de 0.31 hasta $1.94 \%$, pero el subconjunto en donde se establecen la mayor cantidad de 


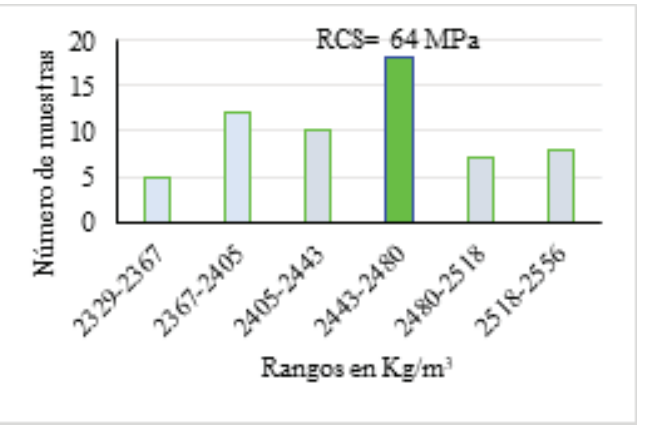

Figure 5

Valores de densidad en mina por rangos.

muestras esta entre 1.12 a $1.40 \%$, y en este rango la RCS presenta en promedio un valor de $61 \mathrm{MPa}$.

De igual manera los datos de absorción en mina presentan un rango de valores de entre 0.32 a 5.99\%, siendo el subconjunto de mayor cantidad de muestras el comprendido entre 0.32 a $1.27 \%$, con lo cual obtenemos del mismo una RCS promedio de $68 \mathrm{MPa}$ (Figura 7).

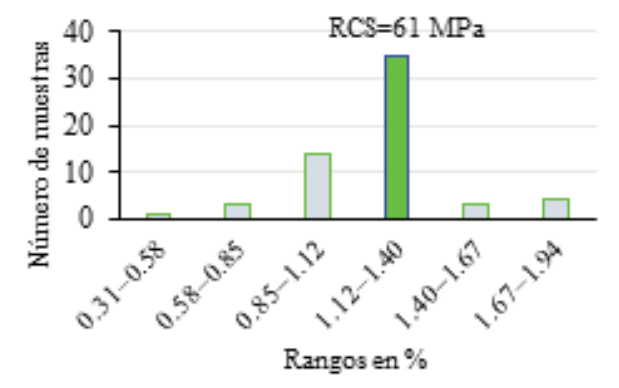

Figure 6

Valores de porosidad en mina por rangos.

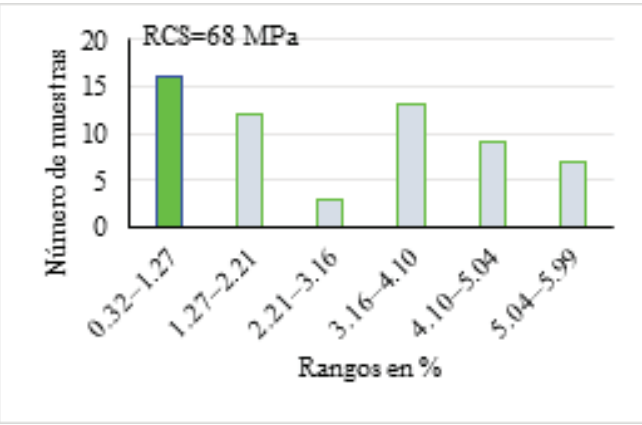

Figure 7

Valores de absorción en mina por rangos.

Dadas las condiciones, debido a los datos analizados, se propone que para la determinacion de las propiedades físicas en mina, descritas anteriormente, se debe 
establecer una metodología de análisis, formando subconjuntos entre densidad y absorción en mina, densidad y porosidad en mina y porosidad y absorción en mina, esto lo podemos observar en la Figura 8 , donde se establecen el número de muestras en cada subconjunto y el promedio en MPa de la RCS.

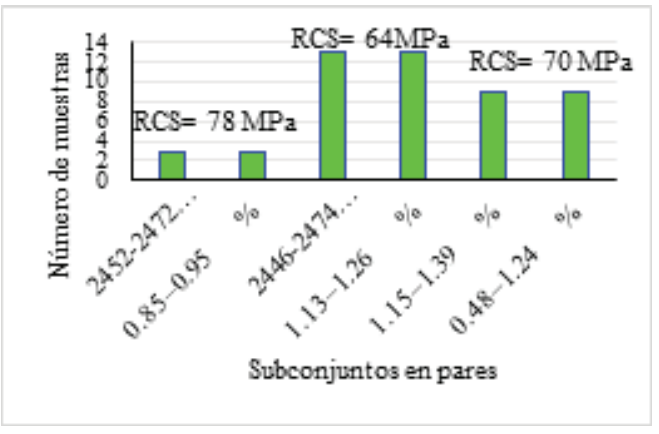

\section{Figure 8}

Muestras por subconjntos dobles.

Finalmente se ha conformado un subconjunto con las muestras que se repiten en las 3 propiedades en mina, lo cual se lo puede observar en la Figura 9, en esta se determina los valores para los rangos de cada una de las propiedades y el promedio de la RCS.

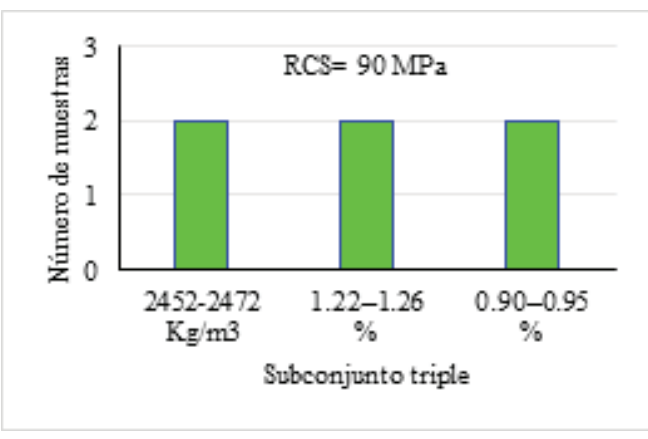

Figure 9

Muestras en subconjunto triple.

\section{Conclusiones}

La metodología planteada nos permite determinar que la roca del Cojitambo tiene una densidad en mina que va desde los 2452 a $2472 \mathrm{Kg} / \mathrm{m}^{3}$, porosidad en mina de entre el 1,22 al 1,26\% y absorción en mina de 0.90 al $0.95 \%$.

Debido a la selección de muestras por medio de subconjuntos, se ha determinado que el valor de la RCS de la roca de Cojitambo tiene en promedio un valor de $90 \mathrm{MPa}$, pero de igual manera si tomamos en consideración los rangos de los subconjuntos, este valor podría estar entre los 61 y $90 \mathrm{MPa}$, lo cual nos ubica siempre dentro de la clasificación como una roca dura. 
Este tipo de roca puede ser usada para elementos ornamentales o de construcción, debido a que su resistencia a compresión simple, mantendría una durabilidad en el tiempo, pero al mismo tiempo permite una maniobrabilidad en el trabajo de destaje.

Una vez evaluados los resultados, que se han obtenido en las experiencias llevadas a cabo, reconocemos el empleo del procedimiento para la valoración de la resistencia a la compresión simple (RCS) de las rocas y caracterizarla por medio de la densidad en mina, porosidad en mina y absorción en mina.

Este trabajo presenta una metodología de trabajo simple y sencillo, para la caracterización de la roca en un proyecto minero, por lo que su puesta a punto debe profundizarse con un mayor número de muestras de diferentes tipos de rocas y de esta forma el proceso sea avalado.

\section{References}

[1] Blyth F, Freitas M. Geología para Ingenieros. México City: México; 2003.

[2] Iriondo M. Introducción a la Geología. Córdova, Argentina; 2006.

[3] Ragan D. Geología Estructural. Barcelona, España; 1980.

[4] Feijoo P, Iñiguez C. Corte en rocas y su relación con la resistencia a compresión simple. Risti [Internet]. 2020 June 5. Available from: http://www.risti.xyz/issues/ristie30.pdf

[5] Feijoo EP, Flores CA, Feijoo BA. The concept of the granulometric area and its relation with the resistance to the simple compression of rocks. Paper presented at: 7th International Engineering, Sciences and Technology Conference (IESTEC); 2019; Panamá. 2019, pp. 52-56, doi: 10.1109/IESTEC46403.2019.00018.

[6] Delgado C. Evaluación de un cambio Tecnológico para el Procesamiento de Minerales de Alta Dureza, Universidad de Chile. 2013.

[7] Galván M. Mecánica de Rocas: Correlación entre la Resistencia a Carga Puntual y la Resistencia a Compresión Simple. Cali, Colombia; 2015.

[8] Secretaria de Comunicaciones y Transporte. Manual de Diseño y Construcción de Túneles de Carreteras. México D. F., México; 2016.

[9] Alonso F. Propiedades Fisicas: Densidad y Porosidad. Universidad de Oviedo; 2013.

[10] Galván M, Restrepo I. Correlación de la resistencia a la compresion uniaxial con la humedad y porosidad eficaz en rocas. Medellín, Colombia: Universidad Nacional de Colombia; 2016.

[11] Ontiveros E, Villegas R. Programa de normalización de estudios previos y control de calidad en las intervenciones. Madrid, España; 1996.

[12] Howland J, Martín A. Estudio de la absorción capilar y la sorptividad de hormigones con áridos calizos Cubanos. Materiales de Construcción. 2013;13

[13] Feijoo P, Román M. Correlación entre la deformación y la resistencia a la compresión en rocas. UCT; 23(91):6.

[14] Feijoo CEP, Padrón SJC. La resistividad de rocas y su relación con la resistencia a compresión simple en mina. uct. 2020;24(99):61-7.

[15] Brito E. Caracterización del material rocoso mediante ensayos físicos de campo [Tesis de Grad]. Cuenca, Ecuador: Universidad del Azuay; 2020.

[16] Rodríguez D, Feijoo P. Determinacion del uso de Plástico PET como alternativa al uso de madera en Fortificación Minera. Lima: CONAMIN; 2018. 